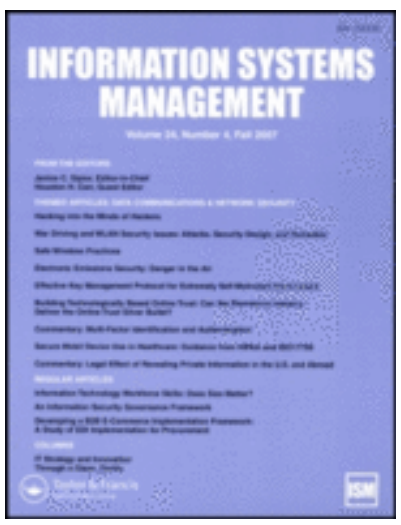

\title{
COVID19 Led Virtualization: Green Data Centre for Information Systems Research
}

\begin{tabular}{|r|l|}
\hline Journal: & Information Systems Management \\
\hline Manuscript ID & UISM-2020-157 \\
\hline Manuscript Type: & Special Issue Manuscript \\
\hline Keywords: & COVID19, virtualization, green information systems, data center \\
\hline
\end{tabular}

\section{SCHOLARONE Manuscripts}


Prof Habin Lee is a chair of Digital Business Analytics at Brunel Business School, Brunel University London. He received a Ph D degree in Management Engineering at KAIST. His research interests include green information systems, computational social science, and virtual communities.

Dr Youngseok Choi is an Associate Professor at Southampton Business School, University of Southampton. He obtained a Ph D degree in Management Information Systems at Seoul National University. His research interests are mainly the application of data analytics to solving managerial problems.

Dr Truong Van Nguyen is a Lecturer at Brunel Business School, Brunel University London. He completed his Ph D study at the Business School, University of Greenwich. His research is centered on application of big data analytics and optimization techniques to supply chain management.

Ms Yang Hai is a Ph D student at Brunel Business School, Brunel University London. She received an MSc degree in Business and Management from University of Liverpool. She is working on simulation optimization for energy efficiency of data centers.

Mr Junchul Kim is a Ph D student at Brunel Business School, Brunel University London. He received an MSc degree in Electronic Telecommunication from Inha University, South Korea. He has a long industrial experience at Korea Telecom before he started his Ph D course. He is doing research on simulation-based analysis of supply chain management focusing on supplier-buyer relationships.

Dr Mohammed Bahja is a Lecturer at Computer Science department at the University of Birmingham. He received a Ph D degree in Computer Science from Brunel University London. His research interests include sentiment analysis and healthcare service quality management.

Prof Hakan Hocaoglu is a chair of Electronic Engineering at Gebze Technical University. He received a Ph D degree from University of Wales. His research interests include power system analysis, power quality and grounding. 


\begin{abstract}
Recent lockdowns over the world due to COVID19 pandemic accelerated virtualization of our social and work life. While virtualization contribute to reducing green-house-gas (GhG) through reduced mobility, it also dramatically increases energy consumption by data centers (DCs) which host large number of servers for realizing virtualization. This paper argues that current engineering-oriented studies on Green DCs needs to be complemented by IS scholars to address human and organizational issues.
\end{abstract}

Key words: COVID19, virtualization, green information systems, data centers. 


\section{Green Virtualization?}

With the COVID19 turning into a pandemic, several million people have been or currently in lockdown all over the world and had to maintain their daily and business life by staying at home. Most of the major companies are still encouraging employees to work remotely. Many countries have switched educational systems online. Virtualization seems now widely penetrating into our lives. The transformation into virtualized life is witnessing the positive impact to environment. According to Guardian, dramatically reduced transportation, operation of factories and offices brought the reduction of carbon emissions by $36 \%$ in the UK.

The green value of IT/IS through virtualized life can be negated if the energy consumed by IT use is great as its saving achieved through its use (Dedrick 2010). The virtualization of work, education, and socialization requires the operation of huge scale of data centers (DCs) which consumes significant amount of energy.

During these unordinary times, DCs have appeared as a lifejacket in terms of sustaining people's lives and governmental systems properly. Because the gigantic number of people spend more time at home than before, the usage of internet and cloud-based applications have been reached an enormous amount that caused overloading the DCs. After the pandemic, it is predicted that people will become more accustomed to doing daily requirements online instead of doing physically, and the explosive demand for small, medium or large scaled DCs will be felt like never before. It probably accelerates the transformation of the world in its existing digitalization.

As the bedrock of digital economy, DCs are among the largest yet growing energy consumers. The energy consumption by DCs world-wide has been already significant before the COVID-19 lockdown. In the US, the total energy consumption by DCs is more than $1.8 \%$ of its total electricity demand in 2014 and the portion is expected to increase by 2020 (Shehabi et al. 2016). Globally, the sector consumes about 3\% of total electricity use (higher than the UK total consumption) and accounts for $2 \%$ of all emissions (on a par with the carbon footprint of the aviation industry). In the EU, DCs use 259 TWh of electricity, which represents $1.7 \%$ of the world's total energy consumption. Due to their significant environment footprint, in Digital Strategy 2020, the EU Commission stresses the urge to make DCs climate-neutral, highly energyefficient and sustainable by no later than 2030.

Over the past decade, despite the rising demand for DC services, the industry's energy consumption was staying nearly flat, as increased Internet traffic and workloads were offset by increased efficiencies of DC operations, including a shift to energy-efficient hyperscale DCs. However, Bashroush and Lawrence (2020) predict that demand for IT services and DCs would substantially outpace efficiency gains over the next five years (2020-2025), resulting in a steady rise in energy use and carbon footprint. Indeed, a widely cited forecast estimates the DC electricity use could increase about 15 -fold by 2030 , to $8 \%$ of the projected global energy demand (Andrae and Edler 2015). Perhaps two underlying drivers for this startling prediction are (1) the fast growth of the computationally intensive cryptocurrency Bitcoin and unprecedented number of people working from home and going online due to the Covid-19 pandemic; and (2) the flattening out and even deterioration of DC efficiency gains in recent years. These drivers indicate that the easiest and most viable efficiency changes have long been fully exploited; therefore, in 
order to achieve the target for climate-neutral DCs by 2030, seeking new energy-saving opportunities is paramount.

\section{Green IT/IS}

Green information systems (Green IS) were noted by scholars since 2000s. The impact of IT/IS have been studied through the first, second, and third-order effects (Dedrick, 2010, Hilty et al 2006; and Kohler and Erdmann 2004). The first order effects include negative environmental issues caused due to the production and use of IT hardware. The manufacturing of computers and the operations of DCs are examples of such effects. The second order effects are outcomes of using IT/IS for transportation and/or industrial production (navigation system reduces the total travel time therefore less green-house-gas (GhG) emissions for example). Finally, the third-order effects are seen from longer term and more dynamic. For example, eBay and Amazon turned our shopping life into virtualized removing travelling to shops.

Existing studies on the Green IS are more focused on the first and second order effects of ISs. Seidel et al (2013) proposes a framework to understand how ISs contribute to the creation of environmentally sustainable organizations and the design of ISs to create required functional affordances. Gholami et al (2018) recently reports how a local business community is using its website to facilitate trading ecofriendly goods and services in West Midlands region of the UK. Seidel et al (2017) reviews the initiative of Green IS and argue that Green IS needs to be integrated into IS research as a core research agenda to improve the sustainability of organizations. The paper also proposes future research agenda through integration with practitioners and different cultures.

There are studies that report positive impacts of adopting Green IS initiative. Nanath and Pillai (2017) also reports the positive impact of Green IS practice that leads to improved green innovation and competitive advantages based on data collected from IT companies in India. Chuang and Huang (2018) confirm that environmental corporate social responsibility (ECSR) facilitates companies to invest on Green ISs assets which leads to positive effects on environmental performance and business competitiveness. Also improved information processing capacities brought by smart grid leads to improved energy efficiency through electricity demand-side management (Corbett 2013).

On the other hand, there are limited studies that provide managers with practical implications to save energy from their business processes. Gawin and Marcinkowski (2017) is one of very few such studies and develop benchmarking scenarios for energy efficient management for retail companies. In this perspective, the role of design scientists is becoming more important to provide practitioners with methods and tools for saving energy. The more design science studies are encouraged in the Green IS literature.

\section{Green DC: Just an Engineering Issue?}

Studies on energy efficient DCs have so far been led by engineers in computer science, electrical engineering, and building engineering. In computer science, developing algorithms for optimal allocation of workloads to servers within DCs has been active research area in the last decade. The algorithms are 
expected to prevent overloading of servers that leads to abnormal consumption of electricity (Dasgupta et al 2011). On the other hand, as the energy consumption by cooling devices sometimes takes more than $50 \%$ of total energy consumption of a DC, thermal management is one of the hot issues in the literature. Scholars apply thermal science and thermal modelling to understand how heats generated by servers are interact with cold air provided by cooling devices (Díaz et al. 2017). The research findings can be applied to designing the location of cooling devices and servers within DC rooms. Exploring an integrated approach to reusing the heat generated from servers as a renewable energy source is another main stream of the Green DC literature (Ding et al. 2019). Scholars in building engineering also investigate the impact of geographical location of DCs to cooling energy saving (Kim et al. 2017). Using simulation tool to identify an optimal design of DC building is another popular approach to energy saving (Pan et al 2008).

On the other hand, government bodies across the world are actively supporting research projects to improve energy efficiency of DCs. EU, for example, have been supporting number of research projects that develop practical solutions for saving energy from DCs. Those projects are aiming to develop energyefficient hardware devices and improve stability, reliability, capability and efficiency of networking equipment, design new switching and routing architecture in order to minimize energy consumption and allocating virtual machines for workload balancing. All projects are generally focused on individual components and provide benefits in terms of its cumulative effects on the DC ecosystem.

Eco4Cloud project ${ }^{1}$ aims to reduce cost, energy consumption and GhG by allocating VM according to energy pricing in DCs at different locations. CATALYST project ${ }^{2}$ on the other hand worked to improve energy efficiency and reuse waste-heat for existing and new DCs. FishDirector project ${ }^{3}$ aims to develop a software that optimizes the use of server resources and minimize energy consumption through optimizing server utilization. BodenTypeDC project ${ }^{4}$ aims to build the most efficient DC that has the PUE value below 1.1 using a fresh air-cooling system and harmonic free renewable energy resources. Finally, GREENDC project ${ }^{5}$ aims at providing a decision support system (DSS) for DC managers by providing monitoring, the estimation and the simulation of energy consumption of DC rooms based on big data obtained from DC room via loT sensors.

\section{Road Ahead for IS scholars}

Unfortunately, studies on DC published on IS journals are very rare. DCs are complex ISs in which IT and cooling devices, human and organizational processes are interacting. While ongoing research for Green DCs in Engineering field is still important, the human and organizational issues for improving energy efficiency of DCs play a pivotal role like other ISs.

Firstly, behavioral issue of energy efficient management of DC needs to be investigated. Identifying factors that facilitate energy efficient behavior in house and work places has been popular topic in Energy discipline (Hori et al 2013). Such studies need to be extended in DC context. One of the traditional

${ }^{1}$ http://www.eco4cloud.com

2 https://project-catalyst.eu/

3 https://www.sardinasystems.com/

${ }^{4}$ https://bodentypedc.eu/

${ }^{5}$ http://www.greendc.eu 
thoughts is that there are some kinds of trade-off relationships between energy consumption and the quality of service (QoS) of DCs. Due to the strategic priority of QoS, DC managers tend to set the indoor temperature of DCs as low as possible to ensure the maximum level of QoS. Though this trend is now being challenged due to advanced technologies for servers and increasing number of DCs are maintaining room temperature higher than before, still the attitude of large number of DC managers is yet to be changed. Studying motivational factors for energy conservative attitude is one of the urgent research issues.

Secondly, the factors and strategies to increase operational efficiency of DCs is another topic for IS scholars. Empirical evidences show that improved operations are among the most-implemented practices for energy efficiency improvement as they do not require substantial investment and avoid service interruption and downtime. Such practices are also the most feasible for small- and medium-size DCs which house the majority of the world's IT equipment and comprise a large fraction of the world's total DC carbon footprint. Current IT operations are inefficient, as more than $66 \%$ of power used by IT in DCs is used to process just $7 \%$ of the work. Hence, the opportunity for energy saving here is enormous but yet underexploited. Decision Support Systems (DSSs) or Expert Support Systems (ESSs) have been widely designed by design scientists in IS discipline and the decision models based on optimization or inference engines of Artificial Intelligence can be applied to maximize energy efficiency of DCs. We need to shift the focus to IT optimization and developing operational strategies that can minimize energy consumption by IT and cooling devices while maintaining the QoS. By this way, our cost-effective and feasible solutions will help substantially increase server utilization (currently as low as $25 \%$ on average and cost $\$ 30$ billion) and provide right-size redundancy level. According to Bashroush and Lawrence (2020), these operational improvements can yield energy savings of up to $90 \%$, bringing us closer to the 2030 target of climateneutral DCs. The specific examples of such operational decision problems include identifying the optimal set points for cooling devices to maintain the room temperature, energy efficient layout of servers and cooling devices, and cost-effective energy sourcing in the consideration of renewable energy sources and traditional power grids.

Thirdly, ISs scholars have been investigating the values of ISs, and the similar studies can be applied to measuring the environmental and economic values of Green DCs. There are number of studies that evaluate the impact of adopting green practices or Green ISs (Nanath and Pillai 2016; Chuang and Huang 2018). While those studies are using survey data for testing cause-effect relationships, econometric models can also be applied to investigate the values of Green DCs using historical data.

\section{Conclusion}

Our lives will never be the same again because of COVID19. Many think that the virus could permanently change the way people work, do shopping and stay open space among others. The dramatic increase of the Internet traffic due to video conferencing, messaging and cloud services will have become 'new normal.' DCs are already the engine of the virtualized lives and Green DCs will be one of the core research agenda of Green ISs. Current engineering efforts to make energy efficient DCs cannot achieve the desired outcomes without collaboration with IS scholars to address the human and organizational issues. The virtualized life will certainly contribute to reducing GhG but at the same time the engine of it needs to be also eco-friendly.

\section{Acknowledgement}


This study was supported by the GREENDC project, which received funding from the European Union's Horizon 2020 research and innovation programme under grant agreement No 734273.

\section{References}

Andrae, A.S. and Edler, T. (2015). On Global Electricity Usage of Communication Technology: Trends to 2030. Challenges, 6(1), 117 - 157. https://doi.org/10.3390/challe6010117.

Bashroush, R. and Lawrence, A. (2020). Beyond PUE: Tackling IT's Wasted Terawatts. UI Intelligence Report, 34. Available at https://uptimeinstitute.com/uptime assets/80ae92ca9b8dfa363a077cb537f51870777499a3921890 6efc6d4e37e28ac3a0-beyond-pue-tackling-its-wasted-terawatts.pdf

Chuang, S.P. and Huang, S.J. (2018). The Effect of Environmental Corporate Social Responsibility on Environmental Performance and Business Competitiveness: The Mediation of Green Information Technology Capital. Journal of Business Ethics, 150, 991-1009.

Corbett, J. (2013). Using Information Systems to Improve Energy Efficiency: Do Smart Meters Make a Difference? Information Systems Frontier, 15, 747 - 760, DOI 10.1007/s10796-013-9414-0.

Dasgupta, G., Sharma, A., Verma, A., Neogi, A. and Kothari, R. (2011). Workload Management for Power Efficiency in Virtualized Data Centers. Communications of the ACM, 54(7), 131 - 141.

Dedrick, J. (2010). GreenIS: Concepts and Issues for Information Systems Research. Communications of the Association of Information Systems, 27(1), $173-184$.

Díaz, A. J., Cáceres, R., Cardemil, J. M., \& Silva-Llanca, L. (2017). Energy and exergy assessment in a perimeter cooled data center: The value of second law efficiency. Applied Thermal Engineering, 124, 820-830. https://doi.org/10.1016/i.applthermaleng.2017.06.066

Ding, Z., Cao, Y., Xie, L., Lu, Y., \& Wang, P. (2019). Integrated Stochastic Energy Management for Data Center Microgrid Considering Waste Heat Recovery. IEEE Transactions on Industry Applications, 55(3), 2198-2207. https://doi.org/10.1109/TIA.2018.2890789

Gawin, B. and Marcinkowski, B. (2017). Business Intelligence in Facility Management: Determinants and Benchmarking Scenarios for Improving Energy Efficiency. Information Systems Management, 34(4), 347 - 358. DOI: 10.1080/10580530.2017.1366219

Gholami, R., Molla, A., Goswami, S., Brewster, C. (2018). Green Information Systems Use in Social Enterprise: The Case of a Community-led Eco-localization Website in the West Midland region of the UK. Information Systems Frontiers, 20, 1345 - 1361.

Hilty, L.M, et al (2006). The Relevance of Information and Communication Technologies for Environmental Sustainability - A Prospective Simulation Study. Environmental Modelling \& Software, 21(11), $1618-1629$. 
Hori, S., Kondo, K., Nogata, D. and Ben, H. (2013). The determinants of household energy-saving behavior: Survey and comparison in five major Asian cities. Energy Policy, 52, $354-362$.

Kim, J. Y., Chang, H. J., Jung, Y. H., Cho, K. M., \& Augenbroe, G. (2017). Energy conservation effects of a multi-stage outdoor air enabled cooling system in a data center. Energy and Buildings, 138, 257270. https://doi.org/10.1016/j.enbuild.2016.12.057

Kohler, A. and L. Erdmann (2004). Expected Environmental Impacts of Pervasive Computing, Human and Ecological Risk Assessment, 10(5), 831-852.

Lossin, F., Kozlovskiy, I., Sodenkamp, M., and Staake, T. (2016). Incentives to go Green: An Empirical Investigation of Monetary and Symbolic Rewards to Motivate Energy Savings. In Proceedings of European Conference on Information Systems (ECIS), Istanbul, Turkey, 12 - 15 June 2016.

Nanath, K. and Pillai, R.R. (2016). The Influence of Green IS Practices on Competitive Advantage: Mediation Role of Green Innovation Performance. Information Systems Management, 34(1), 3-19, DOI: 10.1080/10580530.2017.1254436

Seidel, Stefan; Bharati, Pratyush; Fridgen, Gilbert; Watson, Richard T.; Albizri, Abdullah; Boudreau, Marie-Claude (Maric); Butler, Tom; Kruse, Leona Chandra; Guzman, Indira; Karsten, Helena; Lee, Habin; Melville, Nigel; Rush, Daniel; Toland, Janet; and Watts, Stephanie (2017). The Sustainability Imperative in Information Systems Research. Communications of the Association for Information Systems, 40(3). Available at: http://aisel.aisnet.org/cais/vol40/iss1/3.

Shehabi, Arman, Sarah Smith, Dale Sartor, Richard Brown, Magnus Herrlin, Jonathan Koomey, Eric Masanet, Nathaniel Horner, Inês Azevedo, and William Lintner. (2016). United States Data Center Energy Usage Report. Berkeley, CA (United States). https://doi.org/10.2172/1372902. 